\title{
Bsh coordinates neuronal fate specification with synaptic connectivity
}

\author{
Chundi Xu' ${ }^{1 *}$, Tyler Ramos ${ }^{1}$, Chris Q. Doe ${ }^{1}$ \\ 1'Institute of Neuroscience, Howard Hughes Medical Institute, University of Oregon, Eugene, OR 97403 \\ *Author for correspondence at cxu3@uoregon.edu
}

Key words: neuronal fate, neuronal identity, neuronal diversity, synaptic connectivity, homeodomain transcription factor, feed forward loop, Bsh, Apterous, Zfh1, Pdm3, lamina, DIP- $\beta$

\begin{abstract}
It is widely accepted that neuronal fate is initially determined by spatial and temporal cues acting in progenitors, followed by transcription factors (TFs) that act in post-mitotic neurons to specify their functional identity (e.g. ion channels, cell surface molecules, and neurotransmitters). It remains unclear, however, whether a single TF can coordinately regulate both steps. The five lamina neurons (L1-L5) in the Drosophila visual system, are an ideal model for addressing this question. Here we show that the homeodomain TF Brain-specific homeobox (Bsh) is expressed in a subset of lamina precursor cells (LPCs) where it specifies L4 and L5 fate, and suppresses homeodomain TF Zfh1 to prevent L1 and L3 fate. Subsequently, in L4 neurons, Bsh initiates a feed forward loop with another homeodomain TF Apterous (Ap) to drive recognition molecule DIP- $\beta$ expression, which is required for precise L4 synaptic connectivity. We conclude that a single homeodomain TF expressed in both precursors and neurons can coordinately generate neuronal fate and synaptic connectivity, thereby linking these two developmental events. Furthermore, our results suggest that acquiring LPC expression of a single TF, Bsh, may be sufficient to drive the evolution of increased brain complexity.
\end{abstract}

\section{Main}

The vast diversity of neuron types allows for complex brain function. Each neuron type is generated in a stepwise manner. Transient spatial and temporal cues act in progenitors to specify neuronal fate ${ }^{1-5}$, followed by typespecific TFs, often homeodomain TFs, that act in immature neurons to initiate some or all aspects of neuronal functional identity (e.g. axon/dendrite guidance cues, synaptic specificity molecules, neurotransmitter choice, and ion channels ${ }^{6-10}$. These steps are intimately connected, yet our knowledge of how they are coordinately regulated remains unknown. For example, in both mammals and Drosophila, transient spatial and temporal cues act in progenitors to specify distinct motor neuronal fates $2,3,11$, followed by immature neurons expressing homeodomain TF such as Islet or Nkx6 acting to initiate functional aspects of motor neuron identity ${ }^{12-14}$. This raises important questions: are the TFs that specify initial cell fate always different from the TFs that drive functional aspects of identity? Or are there examples of TFs acting at both steps? Here we choose the Bsh homeodomain TF as a candidate to investigate its potential dual role in neuronal fate specification and subsequent functional identity of lamina neurons in the Drosophila visual system. During late larval and early pupal stages, LPCs give rise to five types of lamina neurons (L1-L5), each with a distinct position in the lamina15. L2/L3 cell bodies are intermingled in the most distal layer while L1, L4, and L5 form distinct layers progressively more proximal (Fig. 1f). Each lamina neuron expresses unique TF markers ${ }^{15}$. 


\section{Bsh is expressed in LPCs and maintained in L4/L5 neurons into the adult}

Lamina neuron development is only partially characterized ${ }^{16-18}$. To better understand how LPCs generate lamina neurons, we identified the Tailless ('Tll) TF as a novel marker for LPCs (Fig. 1a-a"; Extended Data Fig. 1a-a"'). There are two populations of LPCs: cells near the lamina furrow (LF) that express Tll, Homothorax (Hth), and Dac - and cells further from LF that express Tll and Dac but lack Hth (Fig. 1a-a"; Extended Data Fig. 1b-b"'). Importantly, Tll and the neuronal marker Elav have precisely complementary high level expression (Fig. 1a-a”), validating Tll as an LPC marker.

We next addressed the question of whether a TF can be expressed and function in both LPCs and lamina neurons, focusing on homeodomain TFs known to label the L4 (Bsh/Ap) and L5 (Bsh/Pdm3) neurons ${ }^{15}$. We asked whether these TFs were also expressed in LPCs. Bsh was first detected in a subset of LPCs (Fig. 1b-b"'). In contrast, Ap was first detected in L4 neurons (Fig. 1c-c"”, g), and Pdm3 was first detected in L5 neurons (Fig. 1dd"”, g). Ap and Pdm3 were never detected in LPCs or new-born L4 or L5 neurons (Fig. 1c-d"'). Lamina neuron expression of Bsh, Ap and Pdm3 was maintained into the adult (Fig. 1e-e"), consistent with a potential role as terminal selectors in maintaining neuron identity ${ }^{19,20}$. We conclude that Bsh is expressed in both LPCs and neurons, whereas Ap and Pdm3 are expressed only in neurons.

\section{Bsh is required to specify $\mathrm{L} 4 / \mathrm{L} 5$ cell fates}

Bsh is expressed in a subset of LPCs and all L4/L5 neurons, though it is unknown whether L4 and L5 neurons are each generated from a single LPC or two different LPCs. To determine the role of Bsh in LPCs, we used the R27G05-Gal4 driver, which is turned on in all LPCs (Extended Data Fig. 1b-b") to target Bsh RNAi to LPCs. As expected, Bsh-knockdown (KD) eliminates Bsh expression in LPCs and new-born neurons (Extended Data Fig. 2a-b"'). Bsh remains undetectable in the lamina at 3-4 days after pupa formation (3-4d APF) (Fig. 2a-b", quantified in Fig. 2e, f). We next tested whether the absence of Bsh affected L4 and L5 neuronal fate using Ap as an L4 marker and Pdm3 as an L5 marker. We found that Pdm3 was mostly undetectable in the lamina (Fig. 2a-b"', quantified in in Fig. 2g). Similarly, Ap was completely absent in the lamina except a few escaper L5 neurons (Fig. 2c-d"', quantified in in Fig. 2h). Note that the unexpected ectopic Ap in L5 is caused by the R27G05-Gal4 line (Fig. 2c-d"), but is not relevant for our experiments. Furthermore, Bsh Crispr/Cas9 knock out (KO) also led to a loss of Bsh, Ap and Pdm3 from the lamina (Extended Data Fig. 2c-d"'). Taken together, we conclude that Bsh acts in LPCs or newborn neurons to drive expression of Ap and Pdm3 in young neurons and specify L4 and L5 neuronal fates.

To determine if Bsh is sufficient to promote ectopic L4 and L5 neuronal fate, we performed Bshmisexpression in all LPCs. This resulted in significant Bsh expression only in LPCs next to LF but not LPCs further from the furrow (Extended Data Fig. 3a-d") and did not promote ectopic L4 and L5 neuronal fate (Extended Data Fig. 4a-f').

\section{Bsh is required to suppress $\mathrm{L} 1 / \mathrm{L} 3$ neuronal fates}

In the absence of Bsh, what neuronal fate is generated in place of L4 and L5? To test for ectopic generation of another lamina neuron type we assayed for the lamina neuron markers Svp (L1), Bab2 (L2) and Erm (L3). We found that Bsh-KD led to ectopic expression of the L1 and L3 markers Svp and Erm in the positions normally occupied by L4/L5 cell bodies (Fig. 3a-b"”, quantified in Fig. 3i, j). Notably, we never saw the cell bodies co-expressing Erm and Svp, which indicates that the absence of Bsh generates ectopic L1 and L3 
neuron fate but not a hybrid neuronal fate. In contrast, the L2 marker Bab2 was unaffected by Bsh-KD (Extended Data Fig. 5a-b"', quantified in Extended Data Fig. 5c, d). We conclude that Bsh-KD results in ectopic L1/L3 neurons at the expense of L4/L5 neurons.

Although we have shown that Bsh-KD generates L1/L3 neurons instead of L4/L5 neurons, it was not clear if this was a one-to-one switch, e.g. L4>L3 and L5>L1. To identify the neuronal fate generated at the expense of $\mathrm{L} 5$ fate following Bsh-KD, we used Bsh-LexA>LexAop-GFP which labels the small pool of Bsh+ LPCs plus L5 neurons (Extended Data Fig. 5e-f"). We found that Bsh-KD generated GFP+ neurons (normally L5) that lacked the L5 marker Pdm3 and instead showed ectopic expression of the L1 marker Svp (Fig. 3c-d"'); very rarely did we detect the L3 marker Erm (0-1 cell body/optic lobe). Interestingly, some of the GFP+Svp+ neurons had cell bodies displaced from the L5 layer into the L1 layer (Fig. 3d-d"'), which suggests that L1 neurons may actively seek out their appropriate settling layer. We conclude that Bsh is required to specify the L5 neuronal fate and repress L1 fate. This result also allows us to conclude that Bsh-KD results in an L4>L3 switch, as there is no other source for the ectopic L3 neurons.

How does Bsh repress L1 and L3 neuronal fate? Following Bsh-KD, Svp was not detected in the GFP+ LPCs (Extended Data Fig. 5f-g"'). This temporal delay suggests that Bsh acts through an intermediate to repress Svp and Erm expression. To find this intermediate TF, we screened published RNA-seq data ${ }^{15}$ and found only one gene specifically expressed in L1 and L3: Zfh1. We confirmed these results and showed that Zfh1 is present in all LPCs before becoming restricted to L1 and L3 neurons (Extended Data Fig. 6a-b"'). To determine if Zfh1 is required for L1 and L3 neuronal fate, we used RNAi to perform Zfh1-KD in LPCs. As expected, Zfh1-KD eliminates Zfh1expression in all LPCs and neurons (Extended Data Fig. 6e-f'), and Zfh1 remains undetectable at 3-4d APF (Fig. 3e-f"). Importantly, Zfh1-KD resulted in a significant loss of Svp+ L1 and Erm+ L3 neurons (Fig. 3e-f", Extended Data Fig. 6g-h"; quantified in Extended Data Fig. 6i, j). The few remaining might be due to the escape of first-born L1 and L3 neurons prior to maximal Zfh1-KD. Thus, Zfh1 is required for L1 and L3 neuronal fate. In addition, the Bsh-KD resulted in ectopic expression of Zfh1 in the L4/L5 cell body layers (Fig. 3g-h"', quantified in Fig. 3k, l) and in GFP+ neurons (normally L5) (Extended Data Fig. 6c-d"'). We propose that Bsh represses Zfh1 to suppress the L1 and L3 neuronal fate, giving rise to L4 and L5 neuronal fate (Fig. 3o).

\section{Bsh is not required in L4 neurons to maintain L4 neuronal fate}

Bsh is expressed in LPCs and required for specifying L4 fate and inhibiting L3 fate. To test if Bsh expression in L4 neurons is required to maintain L4 neuronal fate, we knocked out Bsh in immature L4 neurons. CRISPR/Cas9 Bsh-KO in L4 neurons resulted in undetectable Bsh protein in the majority of L4 neurons at 2-3d APF (Extended Data Fig. 7a-d). Importantly, we did not observe derepression of L3 markers or any other lamina neuron markers (Extended Data Fig. 8a-h"), nor did we observe loss of Ap expression, showing that Bsh is only required for initiating Ap expression and not for maintaining it (Extended Data Fig. 8e-f"); it is likely that Ap undergoes positive autoregulation after initiation, a common feature of homeodomain $\mathrm{TFs}^{21}$. We conclude that Bsh is not required to maintain L4 neuronal fate.

\section{Bsh/Ap act in feedforward loop to drive the expression of the L4 cell recognition molecule DIP- $\beta$}

Homeodomain TFs often control the acquisition of distinct aspects of neuronal identity ${ }^{8}$. We previously showed that the cell recognition molecule DIP- $\beta$ is specifically expressed in L4 and required for proper L4 circuit connectivity ${ }^{22}$. We asked whether Bsh and/or Ap are required for L4 expression of DIP- $\beta$. We found that Bsh$\mathrm{KO}$ in L4 neurons resulted in the expected loss of Bsh at 2-3d APF (Extended Data Fig. 7a-d) and a strong decrease in DIP- $\beta$ levels (Fig. 4a-b"; quantified in 4a”, b"). Furthermore, using the STaR method ${ }^{22,23}$ we found 
that L4 primary dendrite length and presynaptic Bruchpilot (Brp) puncta in the proximal lamina were both decreased following Bsh-KO (Extended Data Fig. 10a-e), which is distinct from the DIP- $\beta$-KD phenotype ${ }^{22}$. We conclude that Bsh promotes DIP- $\beta$ expression and potentially other cell surface molecules to regulate L4 morphology and connectivity.

To test whether Ap controls DIP- $\beta$ expression in L4, we knocked down Ap using RNAi prior to its time of initiation in L4 neurons (Extended Data Fig. 9a-b"'), and observed a strong decrease in DIP- $\beta$ levels in L4 neurons (Fig. 4c-d"; quantified in Fig. 4c", d"). We next combined the STaR method and Ap-RNAi, and this indeed eliminated Ap expression in L4 at 2d APF (Extended Data Fig. 9c, d) and caused a strong decrease in DIP- $\beta$ levels in L4 neurons (Extended Data Fig. 9e-f"; quantified in Extended Data Fig. 9e", f'). Importantly, Ap-KD in L4 neurons had increased Brp puncta in the distal/proximal lamina and increased length of L4 primary dendrites (Extended Data Fig. 10f-j). This phenotype is similar to the DIP- $\beta-K D$ phenotype 22 , suggesting that Ap might regulate L4 morphology and synaptic connectivity primarily via DIP- $\beta$. Together, our data shows that Bsh activates Ap, and then Bsh and Ap may act together in a feed-forward loop to drive DIP- $\beta$ expression and regulate L4 morphology and synaptic connectivity (Fig. 4e).

\section{Discussion}

Here we show that the Bsh homeodomain TF is expressed in both LPCs and neurons to specify neuronal fate at birth and generate a distinct aspect of neuronal identity: the expression of the cell recognition molecule DIP- $\beta$ which is required for proper neuronal connectivity. Our finding that Bsh regulates neuronal fate is consistent with previous observation that L4 morphology becomes L3-like in Bsh mutant clones ${ }^{24}$, though L3 marker Erm was not used to confirm whether it is a complete cell fate switch.

L1-L3, but not L4 or L5, receive direct synaptic input from the R1-R6 photoreceptors ${ }^{25,26}$ and provide major inputs to the $\mathrm{ON}$ and OFF-pathway circuit (responsive to light increments/decrements) ${ }^{27-30}$. We hypothesize that an ancestral nervous system may have lacked Bsh expression and produced only the core L1-L3 neurons. At some point during evolution, Bsh expression was activated in LPCs, resulting in the suppression of Zfh1 and the formation of L4/L5 neurons; that this increased neuronal diversity could have produced a more complex visual system that was evolutionarily advantageous (Fig. 4e). Consistent with this model, deletion of human BSX (ortholog of Drosophila Bsh) results in Jacobsen syndrome, where patients display cognitive impairment with low IQ, and more than half have ophthalmologic abnormalities ${ }^{31,32}$. Further research on Bsh/BSX function may provide insight into the generation of neuronal diversity in mammals, as well as over an evolutionary time scale.

\section{Acknowledgements}

We thank Stefan Abreo for technical assistance with experiments; Claude Desplan, Lawrence Zipursky, Makoto Sato, Markus Affolter, Richard Mann, Jing Peng, Cheng-Yu Lee; James Skeath, Cheng-Ting Chien for providing antibodies; and Claude Desplan, Vilaiwan Fernandes, Kristen Lee, Emily Heckman and Sarah Ackerman for comments on the manuscript. Stocks obtained from the Bloomington Drosophila Stock Center were used in this study. Funding was provided by HHMI (CQD).

\section{Author Contributions}

CX conceived of the project; performed and analyzed experiments, and generated initial drafts of the text and all figures; TR performed and analyzed experiments, and provided comments on the paper; CQD provided 
feedback during the project, contributed to writing the paper and assembling the figures. All authors commented and approved of the manuscript.

\section{Competing Interest Statement}

The authors declare no competing financial or non-financial interests.

\section{Data Availability Statement}

This study did not generate/analyze datasets/code. Raw data for any main or supplemental figure (.lsm, .czi, or .avi files) can be supplied upon request. 


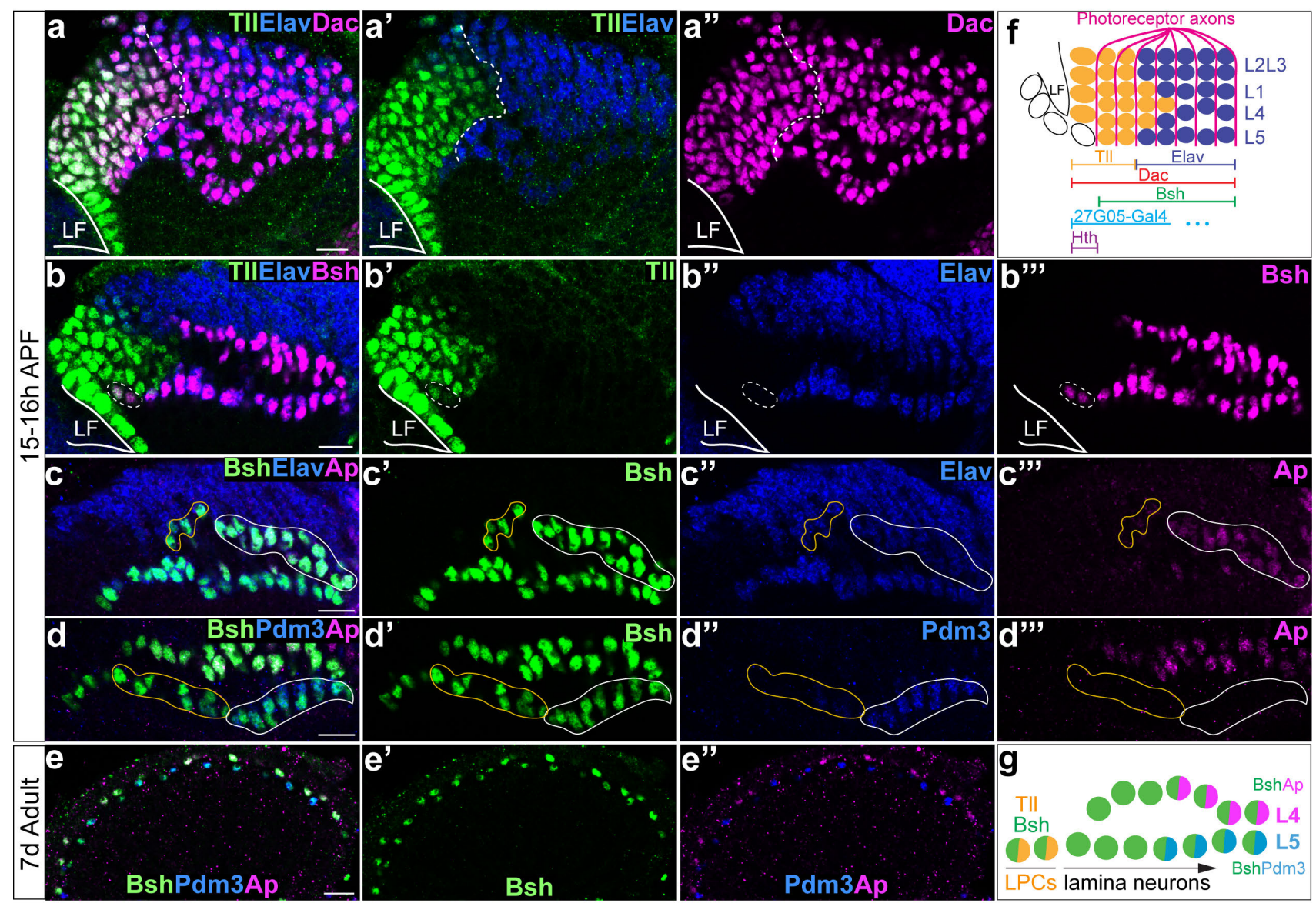

Figure 1: Sequential Expression of Bsh, Apterous and Pdm3 during lamina neuron development.

a-a", Tll is identified as an LPC marker, expressed complementary to Elav; Dac labels both Tll+ LPCs and Elav+ neurons. LF: lamina furrow. Here and below, Scale bar, $10 \mu \mathrm{m} . \mathrm{n}>=5$ brains.

b-b"', Bsh is expressed in Tll+Elav- LPCs (white dotted circle) and maintained in Elav+ L4 and L5 neurons. c-c"', Ap is expressed after the birth of L4 neurons. Newborn L4 neurons are Bsh and Elav positive but Ap negative (yellow line circle). Older L4 neurons are Bsh+ Elav+ Ap+ (white line circle).

d-d"', Pdm3 is first expressed in L5 neurons. Newborn L5 neurons are Bsh+ (yellow line circle), while older L5 neurons are $\mathrm{Bsh}+\mathrm{Pdm} 3+$ (white line circle).

e-e", Bsh and Ap expression is maintained in the adult.

f, Schematic of lamina neuron development in early pupa.

g, Schematic summary. 


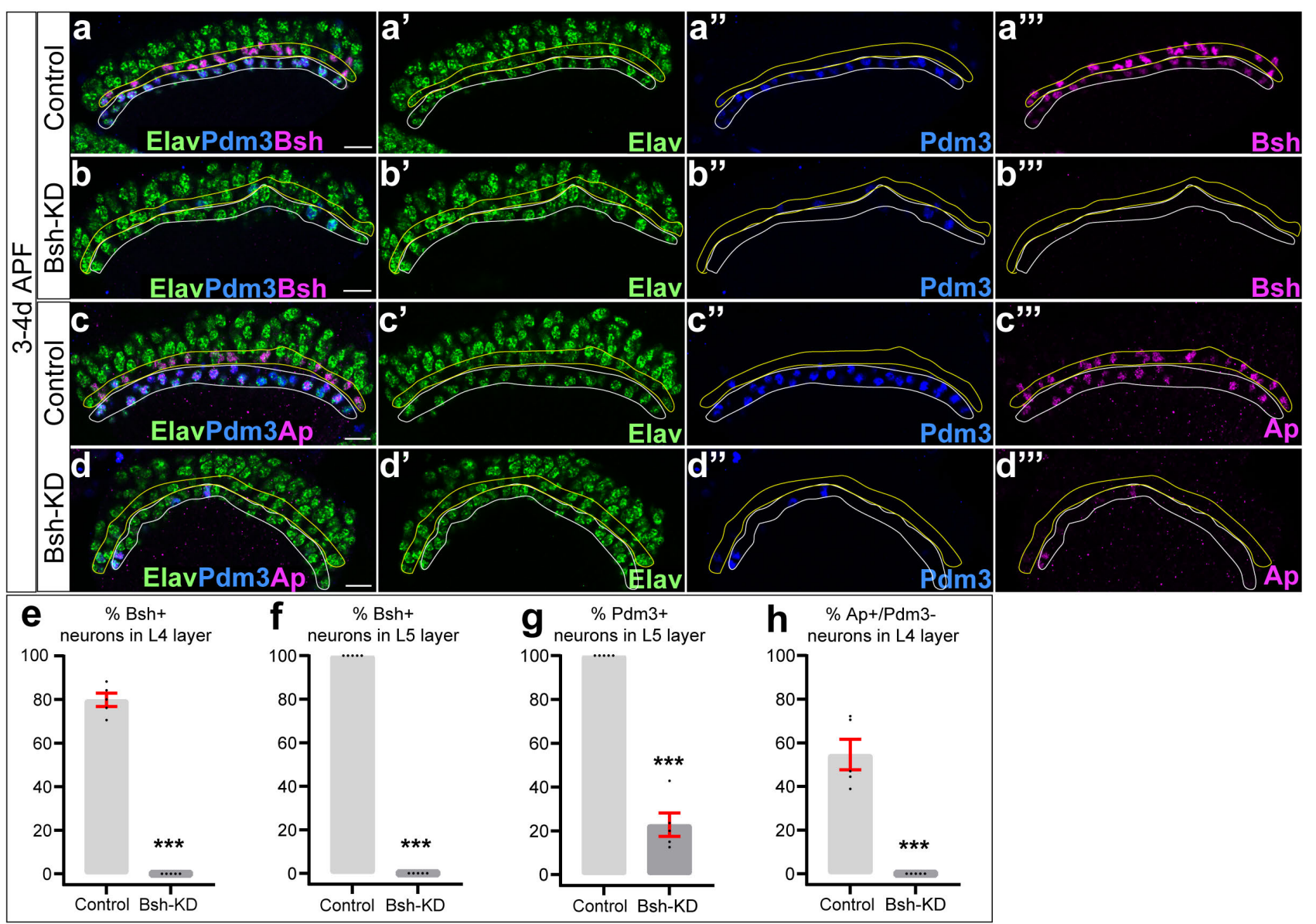

Figure 2: Bsh is required to specify L4/L5 neuronal fates.

a-b"', Bsh KD in LPCs (27G05-Gal4>UAS-Bsh-RNAi) removes most L5 neuron markers (Bsh/Pdm3) from L5 neurons. Here and below, yellow outline, L4 layer; white outline, L5 layer. Scale bar, $10 \mu \mathrm{m}$.

c-d'", Bsh KD in LPCs removes most L4 neuron markers (Bsh/Ap) from L4 neurons. The Ap expression in L5 is caused by the Gal4 driver line but is irrelevant here.

$\mathbf{e}, \mathbf{f}$, The percentage of Bsh+ neurons in L4 layer (yellow outline) (e) and L5 layer (white line) (f).

g, The percentage of Pdm3+ neurons in L5 layer (white outline).

$\mathbf{h}$, The percentage of Ap+/Pdm3- neurons in L4 layer (yellow outline).

Control and Bsh-KD: $\mathrm{n}=5$ brains. $P$-values for each group by unpaired t-test: $P<0.0001$ (Bsh+ in L4 layer), $P<0.0001$ (Pdm3+ in L5 layer), $P<0.0001$ (Ap+/Pdm3- in L4 layer). Data are mean +/- s.e.m. $* * * P<0.0001$. Each dot represents each brain. 


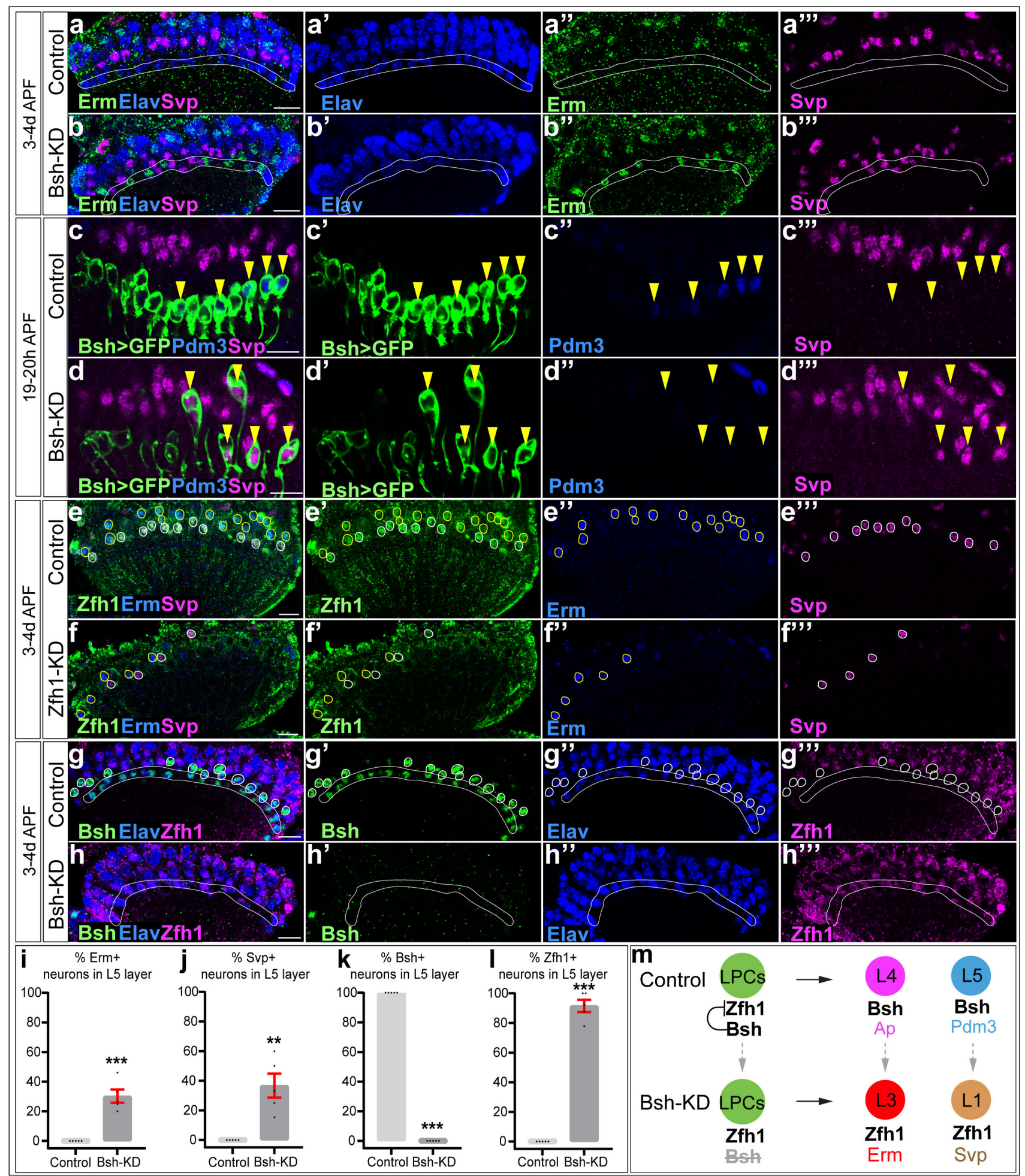

Figure 3: Bsh is required to suppress L1/L3 cell fates.

a-b"”, Bsh KD in LPCs (27G05-Gal4>UAS-Bsh-RNAi) results in ectopic expression of the L1 marker Svp and L3 marker Erm in L4/L5 cell body layers. White outline, L5 layer. Scale bar, $10 \mu \mathrm{m}$.

c-d"”, Bsh KD in LPCs results in ectopic Svp+ L1 neurons, but not Pdm3+ L5 neurons, within Bsh lineage traced L5 layer (Bsh-LexA>LexAop-GFP). Selected Bsh:GFP+ neurons marked with yellow arrowheads. Scale bar, $10 \mu \mathrm{m} . \mathrm{n}>=5$ brains for each group. 
e-f", Zfh1 KD in LPCs results in a loss of Svp+ L1 and Erm+ L3 neurons. Erm + cell bodies are circled with yellow line. Svp + cell bodies are circled with white line. Scale bar, $10 \mu \mathrm{m} . \mathrm{n}>=5$ brains for each group. g-h"”, Bsh KD in LPCs results in ectopic Zfh1 in L4/L5 layers. Bsh+ cell bodies in L4 layer in control are circled with white line. White outline, L5 layer. Scale bar, $10 \mu \mathrm{m}$.

i-1, The percentage of Erm+ neurons (i), Svp+ neurons (j), Bsh+ neurons (k) or Zfh1+ neurons (l) in L5 layer (white outline). Control and Bsh-KD: $\mathrm{n}=5$ brains. $P$-values for each group by unpaired t-test: $P=0.0002($ Erm + in L5 layer), $P=0.002$ (Svp + in L5 layer), $P<0.0001$ ( $\mathrm{Zfh} 1+$ in L5 layer). Data are mean $+/$ - s.e.m. ${ }^{* *} P<0.001$, $* * * P<0.0001$. Each dot represents each brain. m, Summary. 

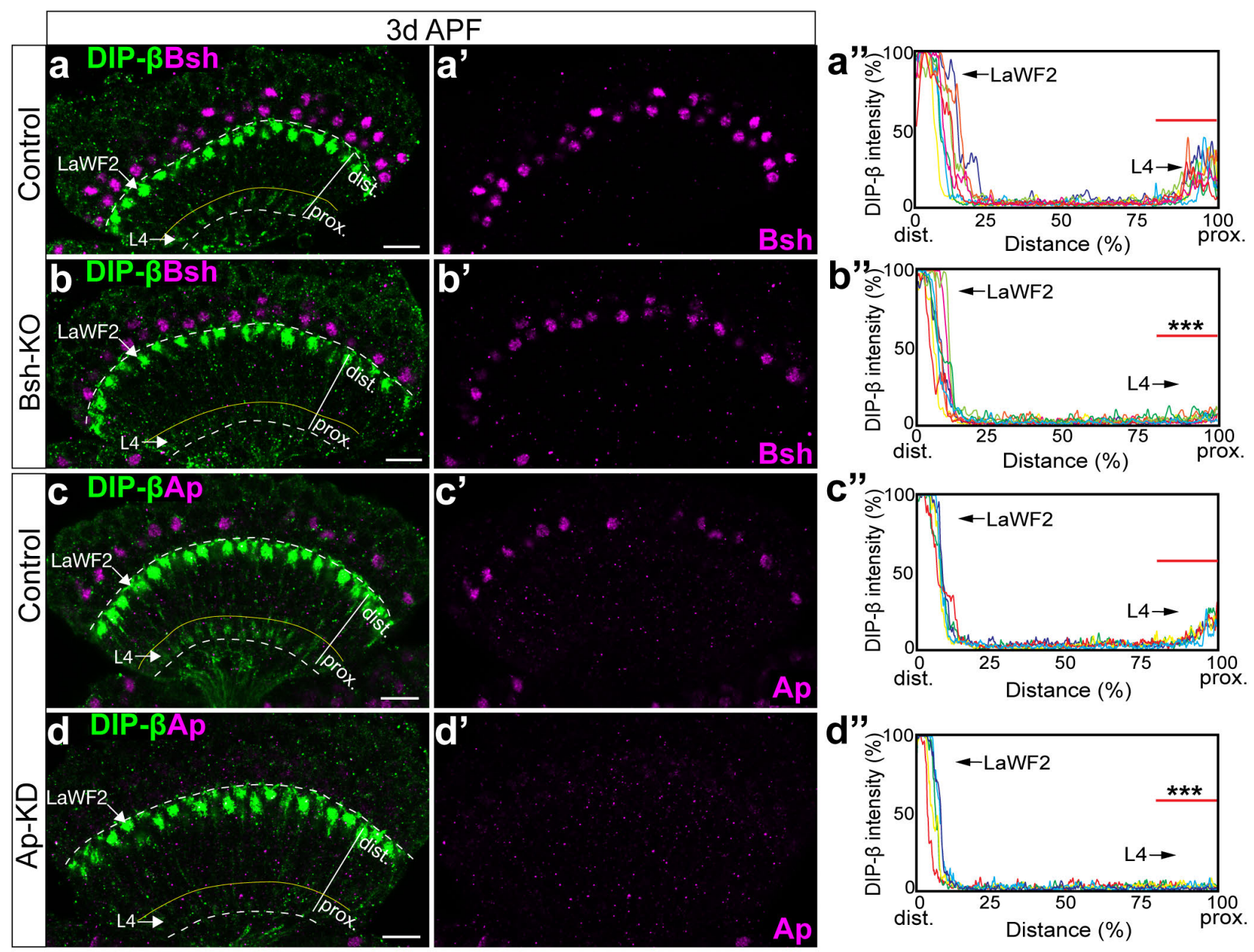

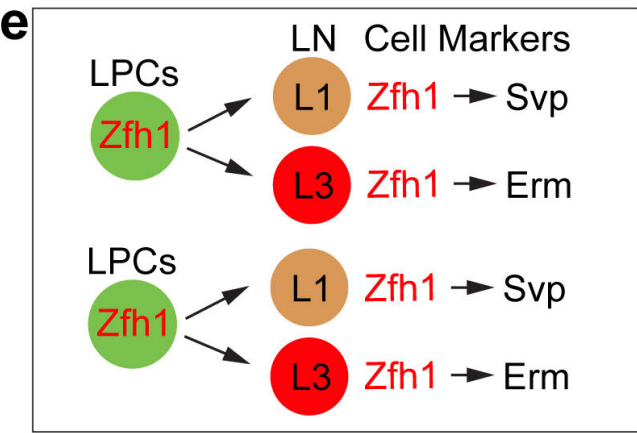

Ancestral function

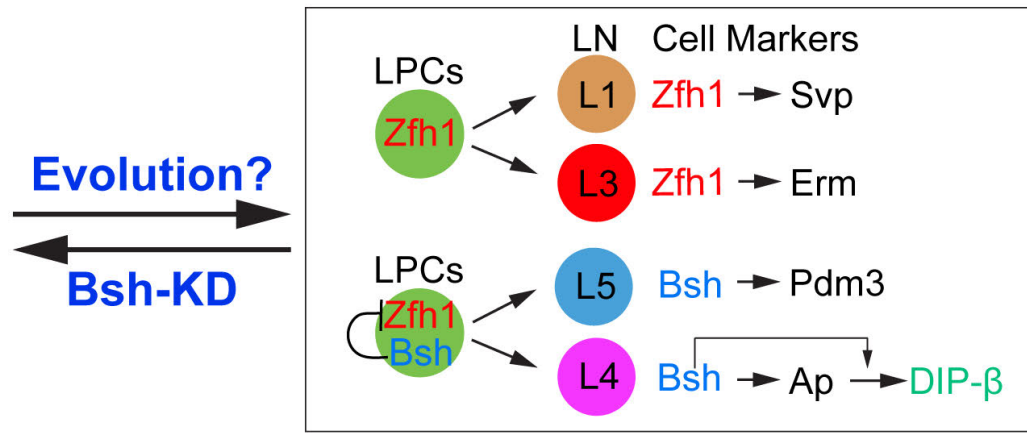

Complex function

Figure 4: Bsh/Ap act in feed forward loop to drive expression of DIP- $\beta$ in L4 neurons.

a-b", Bsh Crispr KO in L4 neurons (31C06AD, 34G07DBD>UAS-Cas9, UAS-Bsh-sgRNAs) results in loss of Bsh and DIP- $\beta$ expression in L4. Note that DIP- $\beta$ signal in the proximal lamina belongs to L4 in control (arrow, L4), whereas DIP- $\beta$ signal in the distal lamina (arrow, likely LaWF2) are not relevant to this experiment. White dotted lines indicate the lamina neuropil; yellow lines show the boundary between the distal and proximal lamina. a", b”, Quantification of DIP- $\beta$ fluorescence intensity along the long axis of lamina cartridges (see white lines in a and b). Significantly reduced fluorescence intensity is observed in the proximal lamina $(80 \%-100 \%$ distance, marked by red bar in a" and b") of Bsh-KO flies compared to Control. Control and Bsh-KO: $\mathrm{n}=3$ cartridges per brain, $\mathrm{n}=8$ brains. $P$-values by unpaired t-test: $P<0.0001$. ${ }^{* * *} \mathrm{P}<0.0001$. Scale bar, $10 \mu \mathrm{m}$. 
c-d", Ap-KD in L4 neurons results in loss of Ap (27G05-Gal4>UAS-ApRNAi) and DIP- $\beta$ expression. c”, d", Quantification of DIP- $\beta$ fluorescence intensity along the long axis of lamina cartridges (see white lines in $\mathrm{c}$ and $\mathrm{d}$ ). Significantly reduced fluorescence intensity is observed in the proximal lamina $(80 \%-100 \%$ distance, marked by red bar in c" and d") of Ap-KD flies compared to Control. Control: $\mathrm{n}=3$ cartridges per brain, $\mathrm{n}=6$ brains. ApKD: $\mathrm{n}=3$ cartridges per brain, $\mathrm{n}=5$ brains. $P$-values by unpaired t-test: $P<0.0001$. $* * * \mathrm{P}<0.0001$. Scale bar, $10 \mu \mathrm{m}$. e, Model. Bsh expression in LPCs increases lamina neuron diversity, and may have evolved to increase neural circuit complexity. Note it is unknown whether L4 and L5 neurons are generated from a single parental LPC or two different LPCs. 

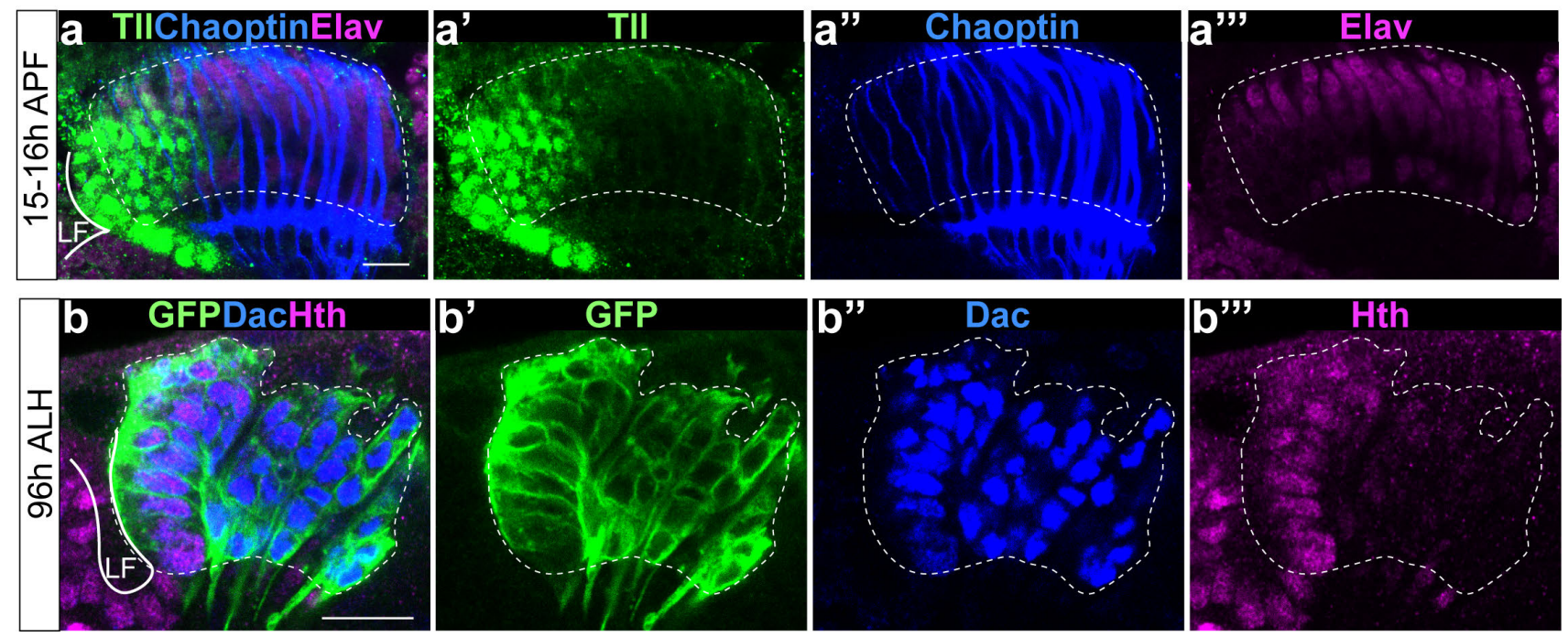

Extended Data Fig. 1: T1l labels LPCs both within the lamina column and before the column, GFP expression driven by 27G05-Gal4 labels all lamina cells at 96 hours after larval hatching.

a-a", Tll+ cells are both within the lamina columns and before the columns. Lamina columns (white dash circle) are within the photoreceptor axons labeled by Chaoptin. Here and below, Scale bar, $10 \mu \mathrm{m}$. $\mathrm{n}>=5$ brains. LF: lamina furrow.

b-b”, 27G05-Gal4 drives GFP expression in all lamina cells (white dash circle) labeled by Dac at 96 hours after larval hatching (ALH). Hth labels LPCs next to lamina furrow ${ }^{17}$. 

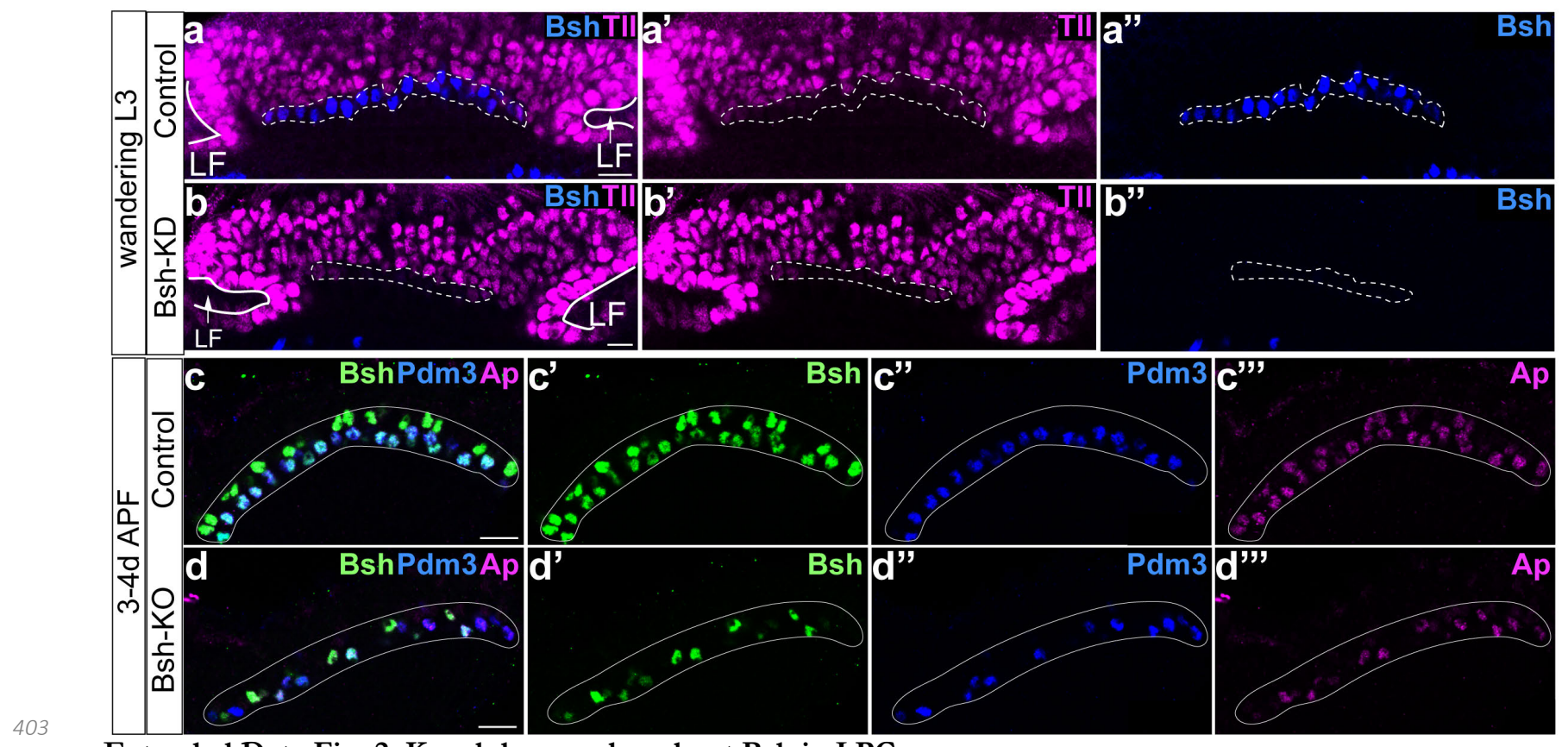

Bsh

Extended Data Fig. 2: Knockdown or knockout Bsh in LPCs.

a-b”, Bsh KD in LPCs (27G05-Gal4>UAS-Bsh-RNAi) eliminates Bsh in LPCs and neurons in wandering L3 larva. Tll labels all LPCs. LF: lamina furrow. L3, third larval instar. Here and below, Scale bar, $10 \mu \mathrm{m} . \mathrm{n}>=5$ brains.

c-d"', Bsh KO in LPCs (27G05-Gal4>UAS-Cas9, UAS-Bsh-sgRNAs) decreases Ap and Pdm3 expression in L4/L5 neurons, respectively. 


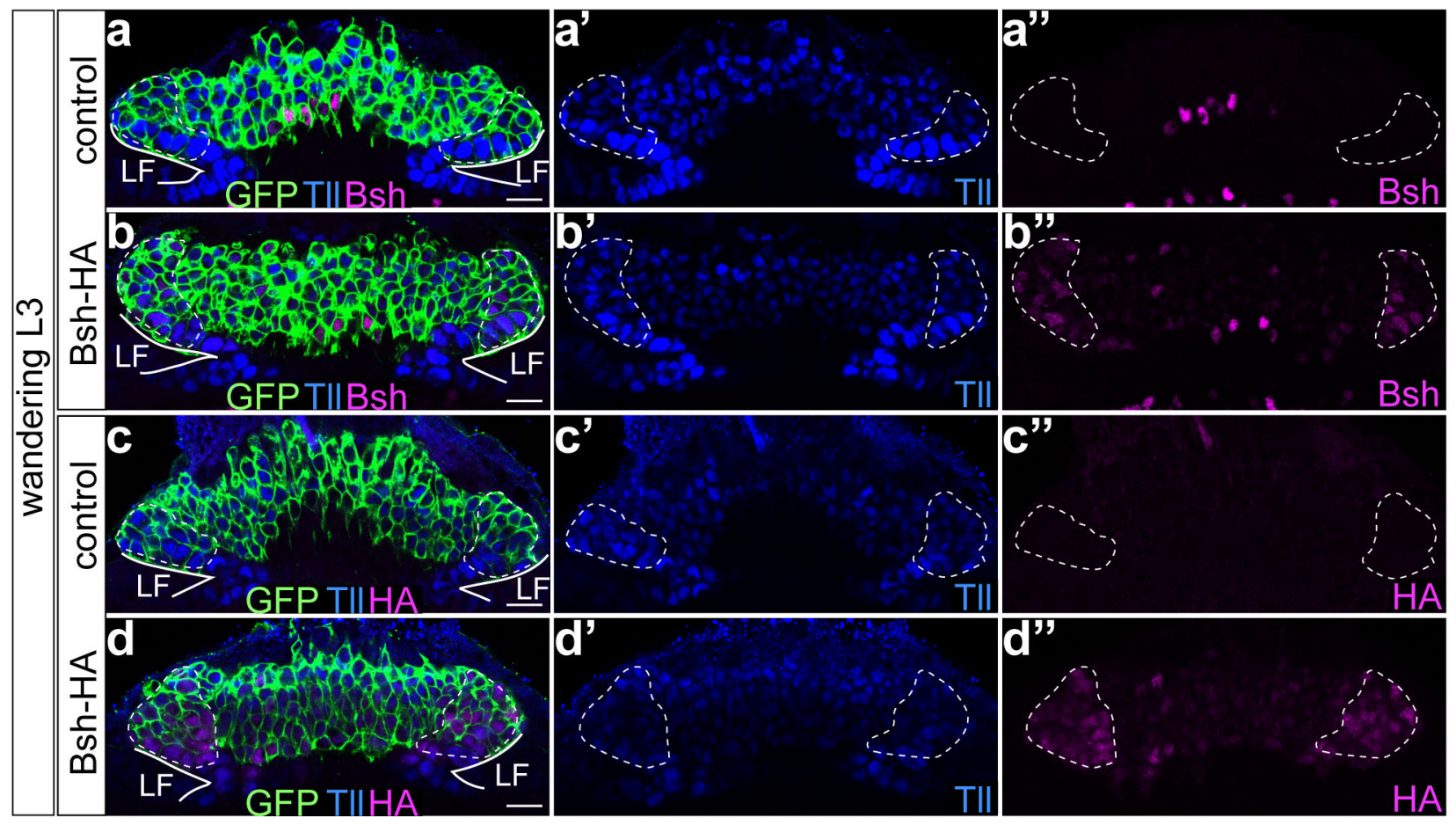

Extended Data Fig. 3: Bsh-HA mis-expression can be detected in a subset of LPCs.

a-b", Bsh-HA mis-expression (27G05-Gal4>UAS-Bsh-HA, UAS-tubP-Gal80[ts]) generates Bsh+ labeling in LPCs next to lamina furrow (white dash circle) in wandering L3 larva. All lamina cells are labeled with GFP (27G05-

Gal4>UAS-myrGFP, UAS-tubP-Gal80[ts]), and Tll labels all LPCs. Here and below, Scale bar, $10 \mu \mathrm{m} . \mathrm{n}>=5$ brains. LF: lamina furrow.

c-d"', Bsh-HA mis-expression generates HA+ labeling in LPCs next to lamina furrow (white dash circle) in wandering L3 larva. All lamina cells are labeled with GFP (27G05-Gal4>UAS-myrGFP, UAS-tubP-Gal80[ts]), and Tll labels all LPCs. 


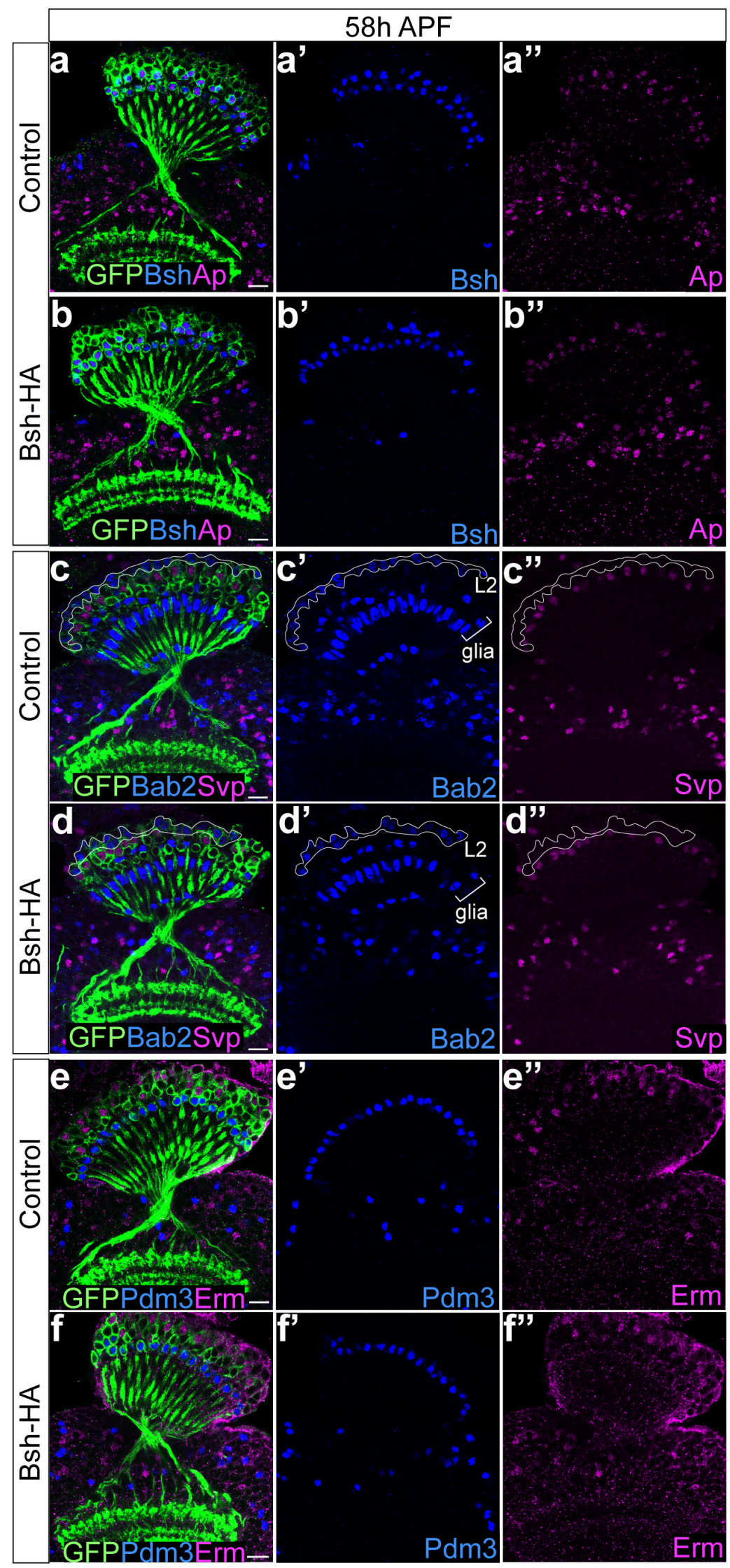


Extended Data Fig. 4: Bsh mis-expression in LPCs next to lamina furrow does not generate ectopic L4/L5 neurons. a-b', Mis-expression of Bsh (27G05-Gal4>UAS-Bsh-HA, UAS-tubP-Gal80[ts]) in furrow-adjacent LPCs does not generate ectopic L4/L5 neurons. The Ap expression in L5 neurons is caused by the Gal4 driver line but is irrelevant here. Here and below, Scale bar, $10 \mu \mathrm{m} . \mathrm{n}>=5$ brains.

c-d", L1 marker Svp and L2 marker Bab2 expressions remain normal in Bsh-mis-expression. e-f”, L3 marker Erm and L5 marker Pdm3 expressions remain normal in Bsh-mis-expression. 


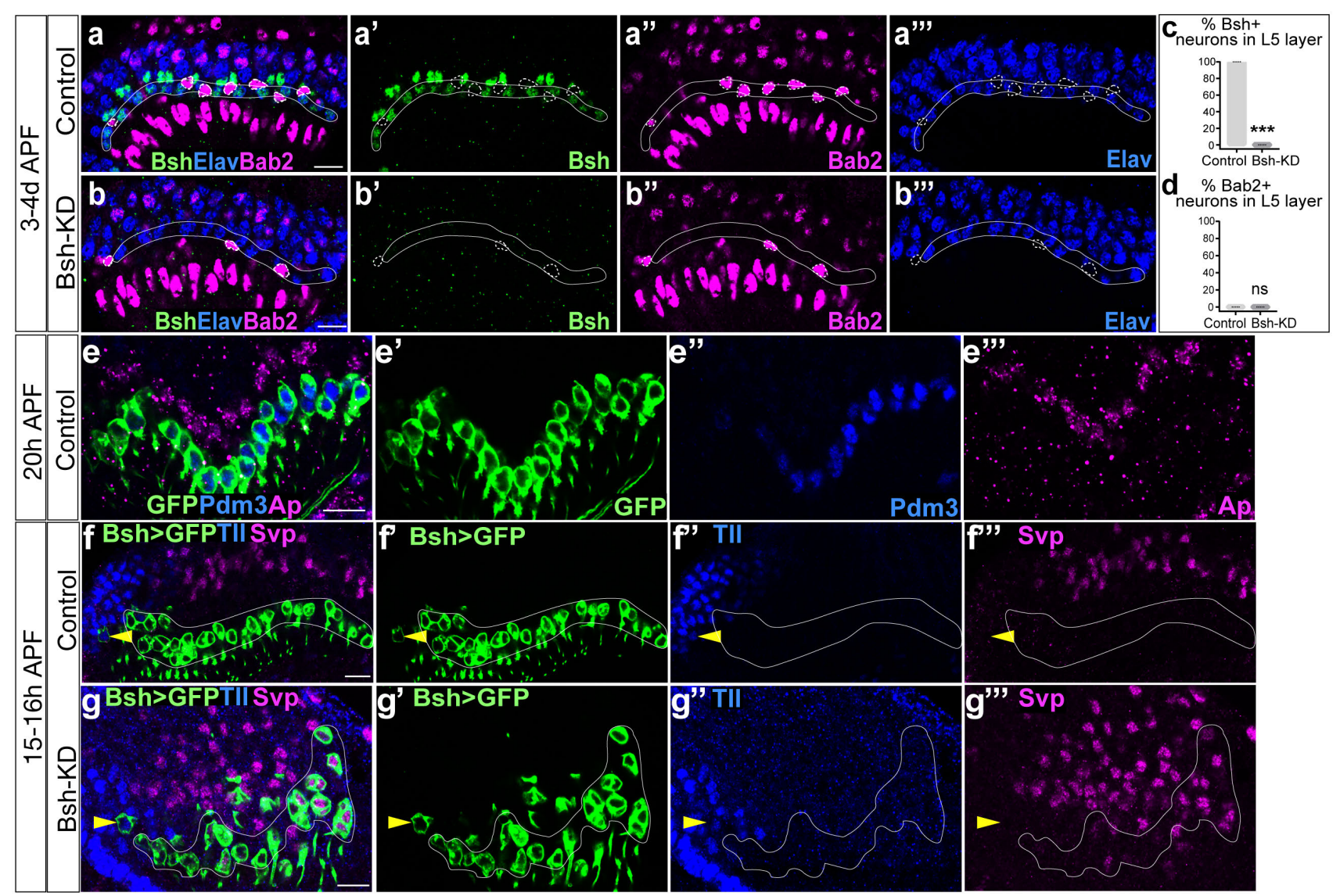

Extended Data Fig. 5: L1 neurons are ectopically generated at the expense of L5 neurons when knocking down Bsh in LPCs.

a-b"', Bsh KD in LPCs (27G05-Gal4>UAS-Bsh-RNAi) does not produce ectopic Bab2-positive L2 neurons. Bsh is absent in Bsh-KD. Bab2-positive cell bodies with white dash circles are glia. White circle labels L5 cell body layer. Scale bar, $10 \mu \mathrm{m}$.

$\mathbf{c}, \mathbf{d}$, The percentage of Bsh+ neurons (c) and Bab2+ neurons (d) in L5 layer (white outline). Control and Bsh-

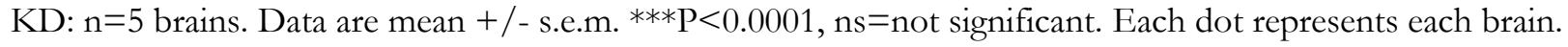
e-e"', Bsh-LexA drives LexAop-myrGFP expression in L5 neurons at 20h APF. Older GFP cell bodies express L5 marker Pdm3. Scale bar, $10 \mu \mathrm{m} . \mathrm{n}>=5$ brains.

f-g'", L1 marker Svp is ectopically expressed in GFP-positive neurons but not GFP-positive LPCs in Bsh-KD. GFP (Bsh-LexA>LexAop-myrGFP) labels LPCs and L5 neurons in control. Tll labels LPCs. White line circle labels GFPpositive neurons. GFP-positive LPCs are Tll positive (yellow arrowhead). Scale bar, $10 \mu \mathrm{m} . \mathrm{n}>=5$ brains. 

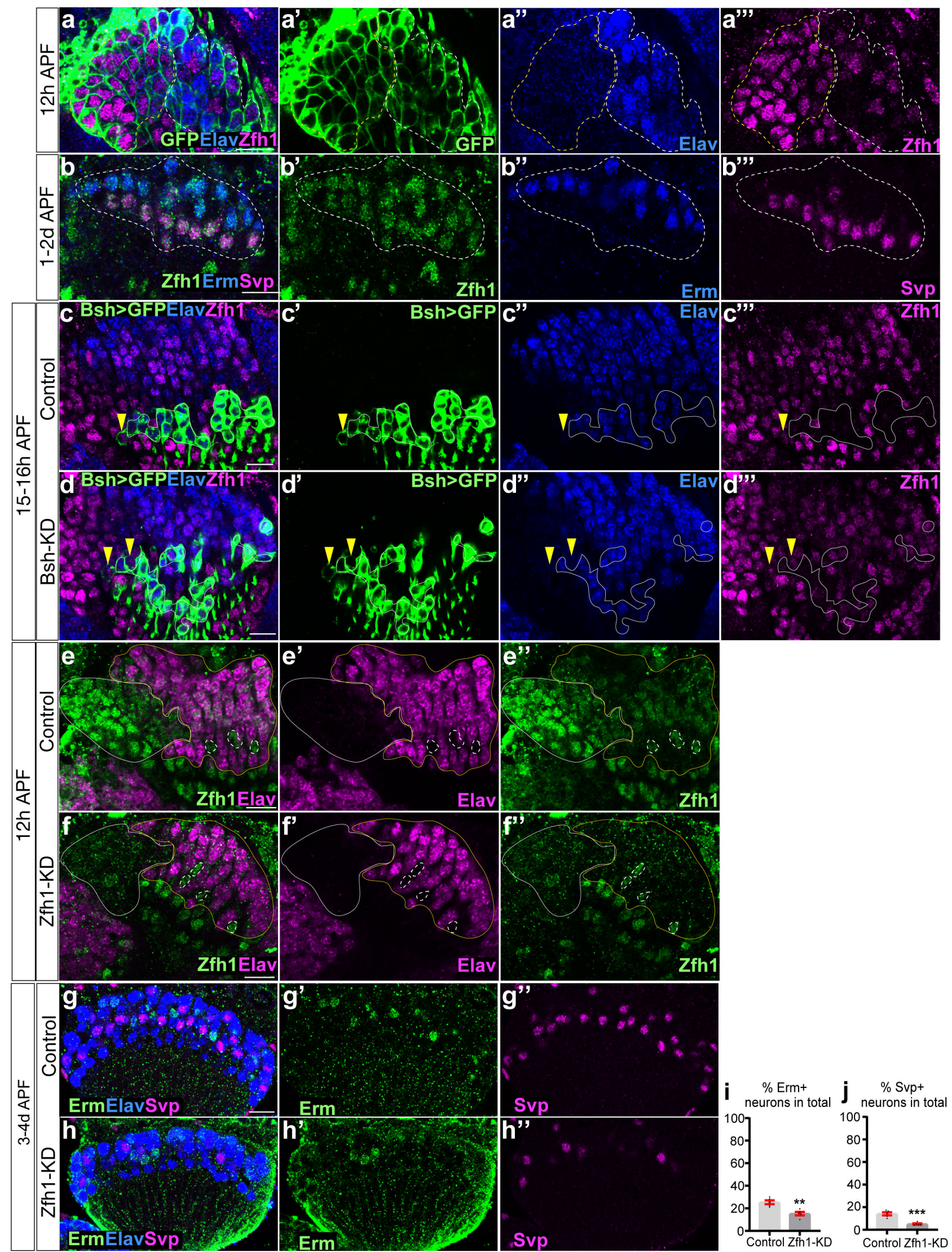
Extended Data Fig. 6: A new L1/L3 marker Zfh1 is expressed in all LPCs, ectopically expressed in Bsh-KD, and Zfh1 knockdown in LPCs eliminates Zfh1 expression in all LPCs and neurons. a-a"', Zfh1 is expressed in all LPCs and some lamina neurons at 12h APF. GFP (27G05-Gal4>UAS-myrGFP) labels all lamina cells. Elav labels lamina neurons. Yellow dash circle outlines LPCs and white dash circle outlines lamina neurons. Scale bar, $10 \mu \mathrm{m} . \mathrm{n}>=5$ brains.

b-b"', Zfh1 is expressed in L1 and L3 neurons at 1-2d APF. Erm labels L3 and Svp labels L1 neurons. White dash circle outlines L1 and L3 neurons. Scale bar, $10 \mu \mathrm{m} . \mathrm{n}>=5$ brains.

c-d"', Zfh1 is ectopically expressed in GFP-positive neurons. GFP (Bsh-LexA>LexAop-myrGFP) labels LPCs and L5 neurons in control. Tll labels LPCs. White line circle outlines GFP-positive neurons. GFP-positive LPCs are Tll positive (yellow arrowhead). Scale bar, $10 \mu \mathrm{m} . \mathrm{n}>=5$ brains.

e-f", Zfh1-KD in LPCs (27G05-Gal4>UAS-Zfh1-RNAi) eliminates Zfh1 expression in all LPCs and lamina neurons at $12 \mathrm{~h}$ APF. White line circle outlines LPCs. Yellow line circle outlines lamina neurons. White dotted circle outlines glia with Zfh1 expression. Scale bar, $10 \mu \mathrm{m} . \mathrm{n}>=5$ brains. g-h", L1 marker Svp and L3 marker Erm are significantly reduced when knocking down Zfh1 in LPCs. Scale bar, 10 $\mu \mathrm{m}$.

$\mathbf{i}, \mathbf{j}$, The percentage of Erm + neurons (i) and Svp + neurons (j) in total lamina neurons. Control and Zfh1-KD: $\mathrm{n}=5$ brains. $P$-values for each group by unpaired t-test: $\mathrm{P}=0.0023$ (Erm + total), $\mathrm{P}=0.0003$ (Svp + total). Data are mean + /- s.e.m. ${ }^{* * P} P 0.001,{ }^{* * *} P<0.0001$. Each dot represents each brain. 


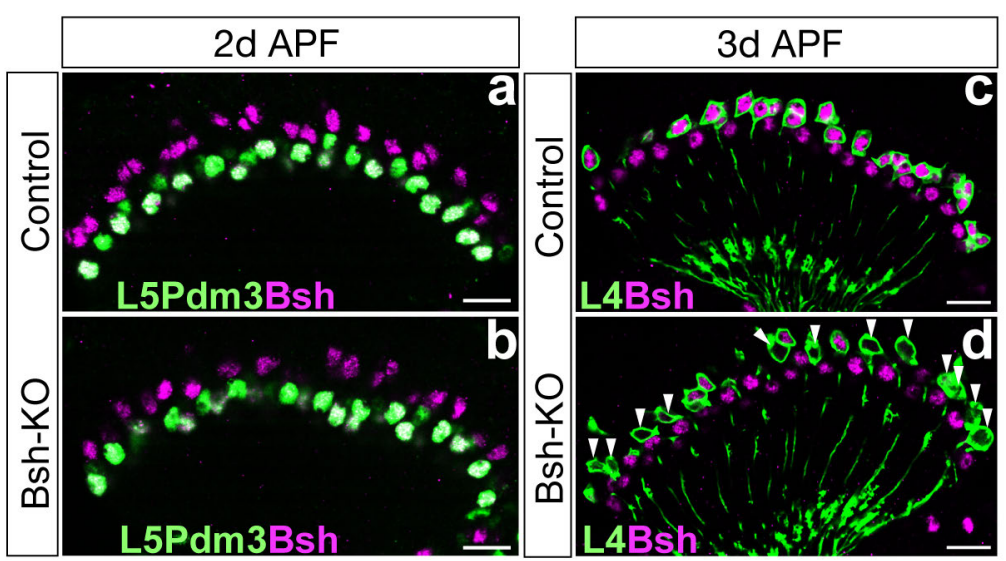

Extended Data Fig. 7: Knockout of Bsh in L4 neurons results in Bsh loss between 2d and 3d APF.

a, b, Bsh expression remains normal in L4 neurons when knockout Bsh in L4 neurons (31C06AD,

34G07DBD>UAS-Cas9, UAS-Bsh-sgRNAs) at 2d APF. Bsh-positive and Pdm3-negative labels L4 cell bodies. Here and below, Scale bar, $10 \mu \mathrm{m} . \mathrm{n}>=5$ brains.

$\mathbf{c}, \mathbf{d}$, Bsh expression is absent in most L4 neurons when knockout Bsh in L4 neurons (31C06AD, 34G07DBD>UASCas9, UAS-Bsh-sgRNAs) at 3d APF. GFP (31C06AD, 34G07DBD>UAS-myrGFP) labels L4 neurons. White arrowhead indicates L4 cell body lacking Bsh expression. 


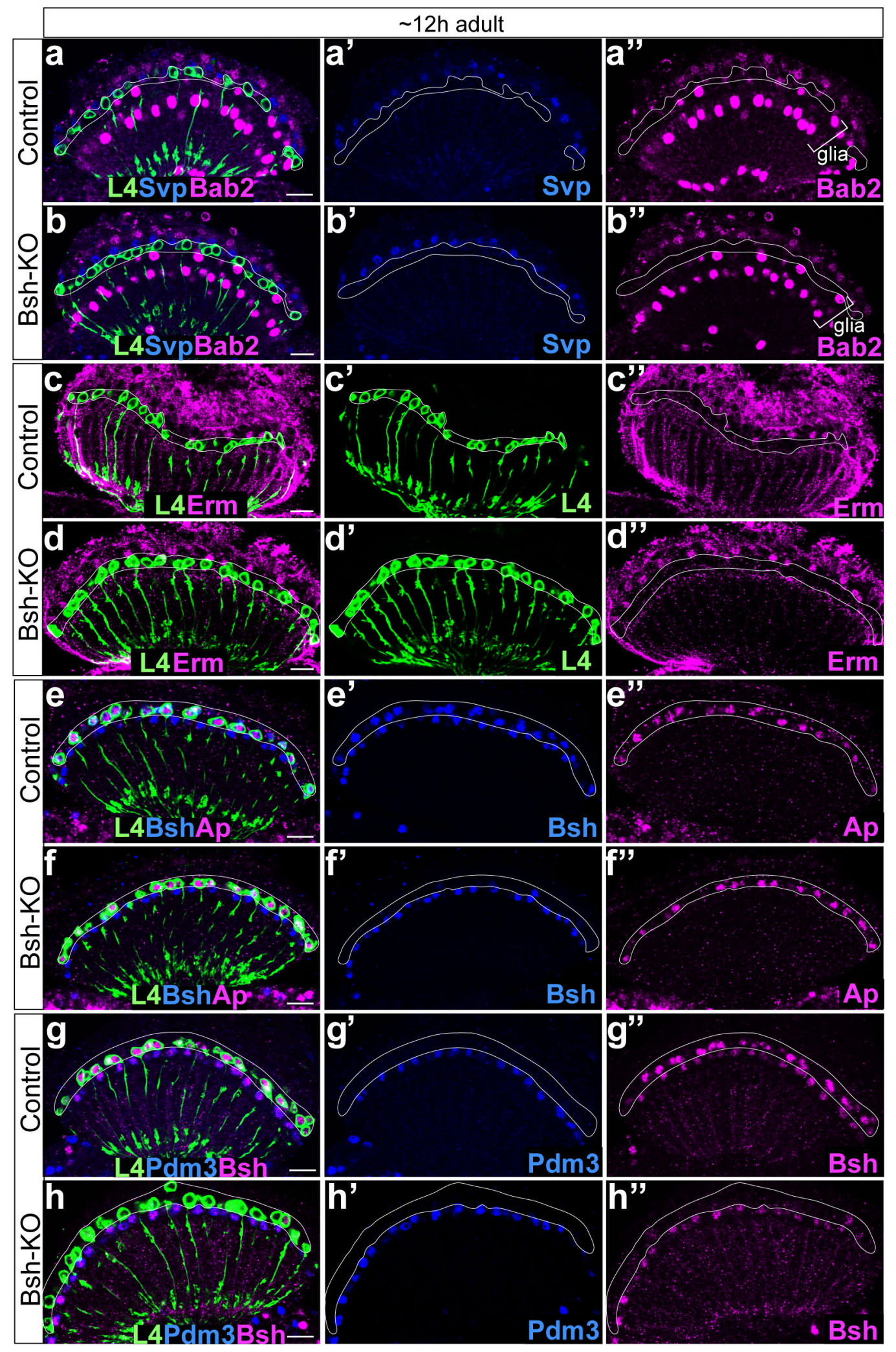

Extended Data Fig. 8: Bsh is not required in neurons to maintain L4 neuronal fate. 
a-b", L1 marker Svp and L2 marker Bab2 are not detected in L4 cell bodies when knocking out Bsh in L4 (31C06AD, 34G07DBD>UAS-Cas9, UAS-Bsh-sgRNAs). Here and below, L4 is labeled by GFP (31C06AD, 34G07DBD> UAS-myrGFP). White outline, $\mathrm{L} 4$ cell body. Scale bar, $10 \mu \mathrm{m} . \mathrm{n}>=5$ brains. c-d", L3 marker Erm is not detected in L4 cell bodies when knocking out Bsh in L4.

e-f", Bsh becomes absent in most L4 while Ap expression remains normal when knocking out Bsh in L4. g-h", L5 marker Pdm3 is not detected in L4 cell bodies when knocking out Bsh in L4. 


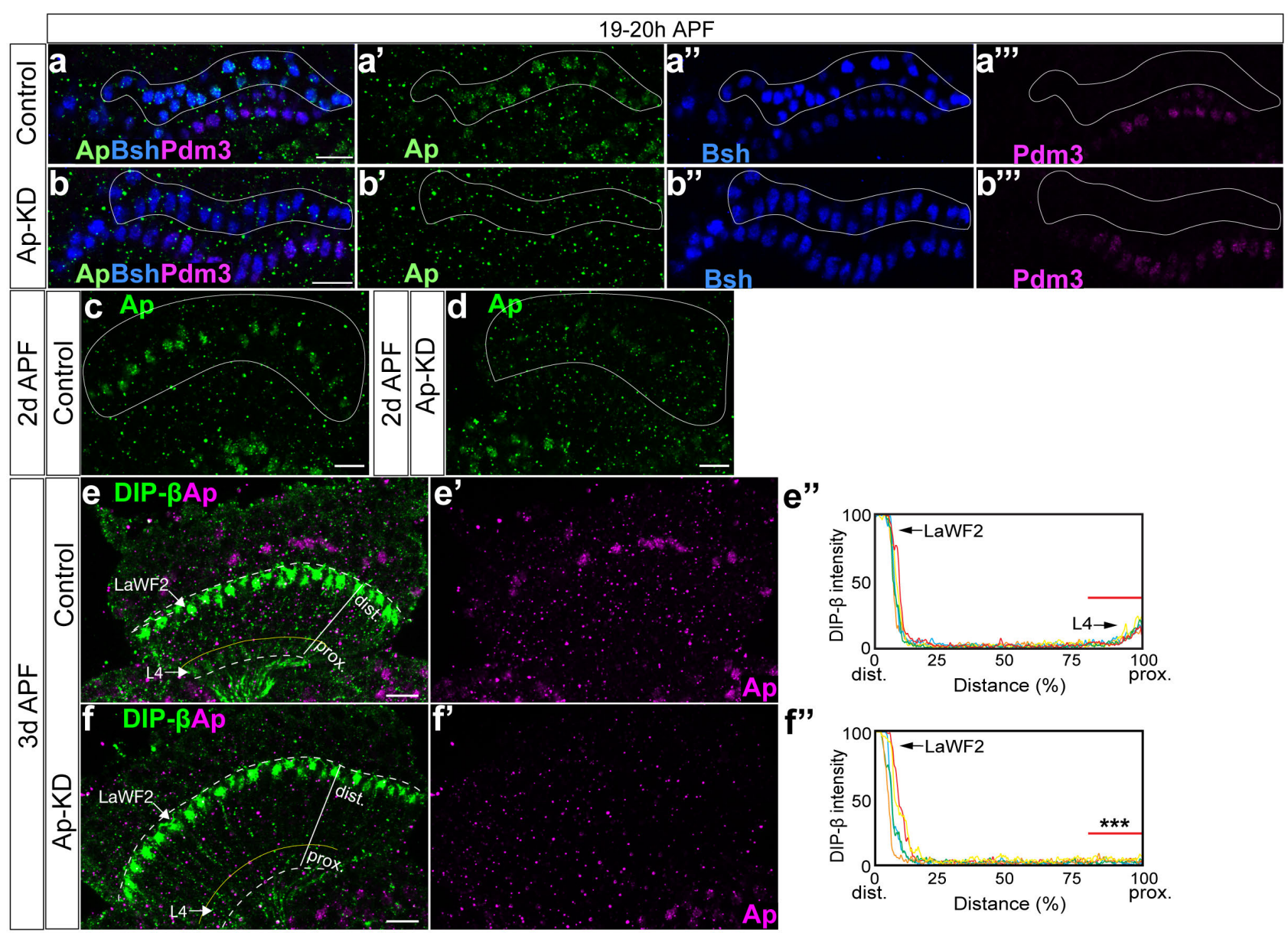

Extended Data Fig. 9: DIP- $\beta$ expression is disrupted when knocking down Ap in L4 neurons. circle outline L4 cell bodies (top row of Bsh positive). Pdm3 is expressed in early born L5 neurons (bottom row of Bsh positive). The Ap expression in L5 neurons is caused by the Gal4 driver line but is irrelevant here. Scale bar, $10 \mu \mathrm{m}$. $\mathrm{n}>=5$ brains.

c, d, Ap-KD (31C06AD, 34G07DBD>UAS-RSR and 79C23S-GS-rst-stop-rst-smFPV5-2a-GAL4 and UAS-ApshRNA) eliminates Ap expression in L4 neurons at 2d APF. White line circle outlines lamina neuron cell bodies. Scale bar, $10 \mu \mathrm{m} . \mathrm{n}>=5$ brains.

e, f', DIP- $\beta$ expression is disrupted in L4 neurons in Ap-KD (31C06AD, 34G07DBD>UAS-RSR and 79C23S-GSrst-stop-rst-smFPV5-2a-GAL4 and UAS-Ap-shRNA). White dotted lines indicate the lamina neuropil. The yellow lines show the boundary between the distal and proximal lamina. Note that DIP- $\beta$ signal in the proximal lamina belongs to L4 in control (arrow, L4), whereas DIP- $\beta$ signal in the distal lamina (arrow, likely LaWF2) are not relevant to this experiment. e", f", Quantification of DIP- $\beta$ fluorescence intensity along the long axis of lamina cartridges (see white lines in e and f). Significantly reduced fluorescence intensity is observed in the proximal lamina (80\%-100\% distance, marked by red bar in e" and f') of Ap-KD flies compared to Control. Control and Ap-KD: n=3 cartridges per brain, $\mathrm{n}=5$ brains. $P$-values by unpaired t-test: $P<0.0001$. ${ }^{* * *} \mathrm{P}<0.0001$. Scale bar, $10 \mu \mathrm{m}$. 


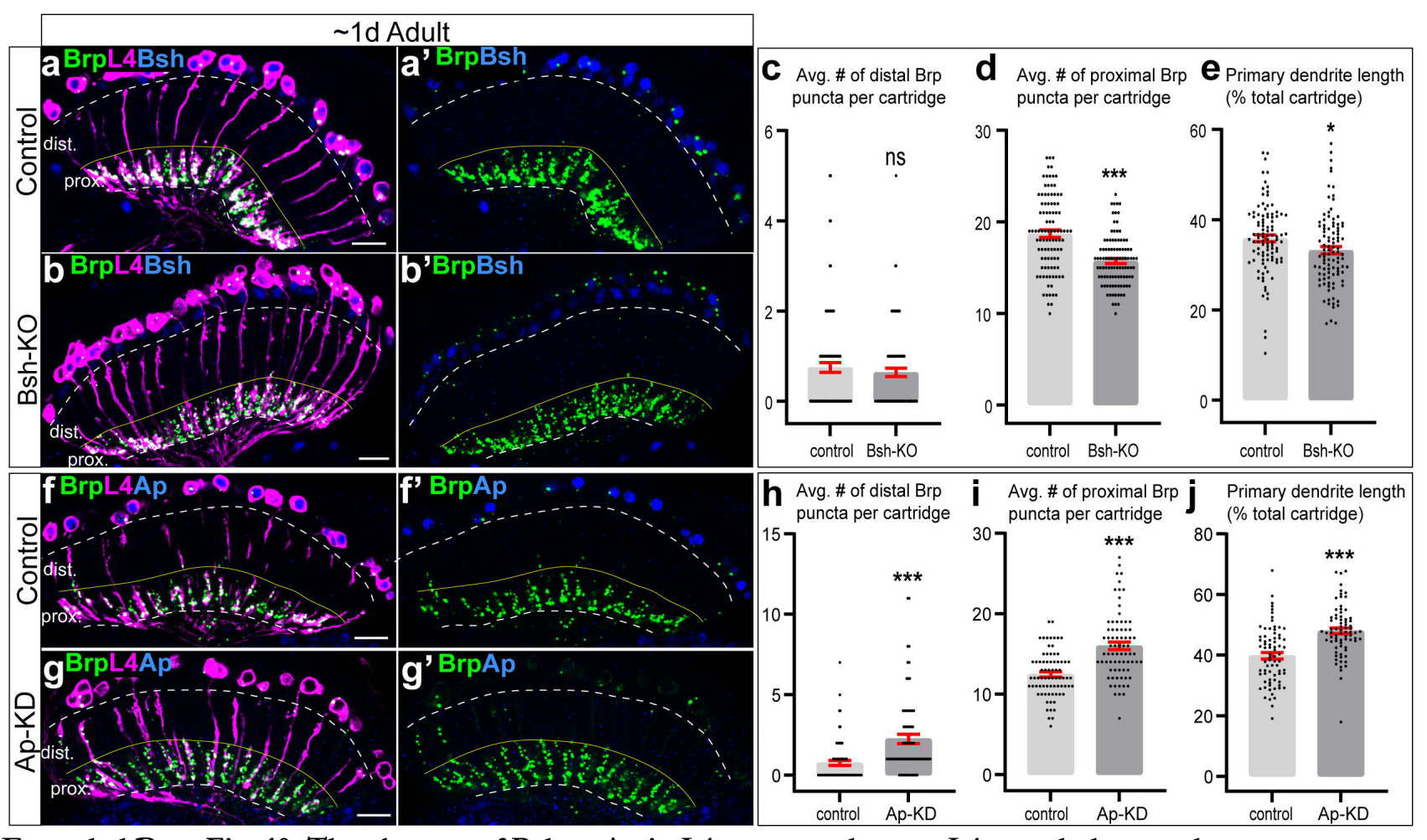

Extended Data Fig. 10: The absence of Bsh or Ap in L4 neurons changes L4 morphology and synapse number.

a-e, Bsh knockout decreases primary dendrite length and proximal synapse number in L4 (31C06AD, 34G07DBD>UAS-Cas9, UAS-Bsh-sgRNAs, UAS-myrGFP, UAS-RSR, Brp-rst-stop-rst-smFPV5-2a-GAL4). a-b', Bsh expression is absent in most L4 neurons in Bsh-KO. White dotted lines indicate the lamina neuropil. The yellow lines show the boundary between the distal and proximal lamina. $\mathbf{c}, \mathbf{d}$, the average number of Brp puncta in L4 neurons present within the distal (c) or proximal (d) halves of lamina cartridges. e, L4 primary dendrite length calculated as percentage of total cartridge length. $c$, $d$ and e: control: $n=100$ cartridges, $n=5$ brains; Bsh-KO: $n=100$ cartridges, $\mathrm{n}=5$ brains. $P$-values for each group by unpaired t-test: $P=0.4464$ (distal brp), $P<0.001$ (proximal brp), $P=0.0198$ (proximal neurites). Data are mean $+/$ - s.e.m. $* P<0.01$, $* * * P<0.0001$. Each dot represents each cartridge.

$\mathbf{f}-\mathbf{j}$, Ap knockdown increases L4 primary dendrite length and synapse number in L4 (31C06AD, 34G07DBD>UASRSR, 79C23S-GS-rst-stop-rst-smFPV5-2a-GAL4, UAS-Ap-shRNA, UAS-myrGFP). Ap expression is absent in L4 neurons in Ap-KD. White dotted lines indicate the lamina neuropil. The yellow lines show the boundary between the distal and proximal lamina. $\mathbf{h}, \mathbf{i}$, the average number of Brp puncta in L4 neurons present within the distal (h) or proximal (i) halves of lamina cartridges. j, L4 primary dendrite length calculated as percentage of total cartridge length. h, $i$ and j: control: $n=100$ cartridges, $n=5$ brains; Bsh-KO: $n=100$ cartridges, $n=5$ brains. $P$-values for each group by unpaired t-test: $P<0.0001$ (distal brp), $P<0.001$ (proximal brp), $P<0.0001$ (proximal neurites). Data are mean $+/-$ s.e.m. ${ }^{* * *} P<0.0001$. Each dot represents each cartridge. 


\section{References}

1. Erclik, T. et al. Integration of temporal and spatial patterning generates neural diversity. Nature 541, 365-370 (2017).

2. Doe, C. Q. Temporal Patterning in the Drosophila CNS. 24 (2017).

3. Sen, S. Q., Chanchani, S., Southall, T. D. \& Doe, C. Q. Neuroblast-specific open chromatin allows the temporal transcription factor, Hunchback, to bind neuroblast-specific loci. eLife 8, e44036 (2019).

4. Holguera, I. \& Desplan, C. Neuronal specification in space and time. Science 362, 176-180 (2018).

5. Bayraktar, O. A. \& Doe, C. Q. Combinatorial temporal patterning in progenitors expands neural diversity. Nature 498, 449-455 (2013).

6. Reilly, M. B., Cros, C., Varol, E., Yemini, E. \& Hobert, O. Unique homeobox codes delineate all the neuron classes of C. elegans. Nature 584, 595-601 (2020).

7. Cubelos, B. et al. Cux1 and Cux2 Regulate Dendritic Branching, Spine Morphology, and Synapses of the Upper Layer Neurons of the Cortex. Neuron 66, 523-535 (2010).

8. Hobert, O. \& Kratsios, P. Neuronal identity control by terminal selectors in worms, flies, and chordates. Curr. Opin. Neurobiol. 56, 97-105 (2019).

9. Enriquez, J. et al. Specification of Individual Adult Motor Neuron Morphologies by Combinatorial Transcription Factor Codes. Neuron 86, 955-970 (2015).

10.Hasegawa, E., Kaido, M., Takayama, R. \& Sato, M. Brain-specific-homeobox is required for the specification of neuronal types in the Drosophila optic lobe. Dev. Biol. 377, 90-99 (2013).

11.Holguera, I. \& Desplan, C. Neuronal specification in space and time. BRAIN Dev. 5 (2018).

12.Thor, S. \& Thomas, J. B. The Drosophila islet Gene Governs Axon Pathfinding and Neurotransmitter Identity. Neuron 18, 397-409 (1997).

13.De Marco Garcia, N. V. \& Jessell, T. M. Early Motor Neuron Pool Identity and Muscle Nerve Trajectory Defined by Postmitotic Restrictions in Nkx6.1 Activity. Neuron 57, 217-231 (2008).

14.D’Elia, K. P. \& Dasen, J. S. Development, functional organization, and evolution of vertebrate axial motor circuits. Neural Develop. 13, 10 (2018). 
15.Tan, L. et al. Ig Superfamily Ligand and Receptor Pairs Expressed in Synaptic Partners in Drosophila. Cell 163, 1756-1769 (2015).

16.Huang, Z., Shilo, B. Z. \& Kunes, S. A retinal axon fascicle uses Spitz, an EGF receptor ligand, to construct a synaptic cartridge in the brain of Drosophila. Cell 95, 693-703 (1998).

17.Piñeiro, C., Lopes, C. S. \& Casares, F. A conserved transcriptional network regulates lamina development in the Drosophila visual system. Dev. Camb. 141, 2838-2847 (2014).

18.Fernandes, V. M., Chen, Z., Rossi, A. M., Zipfel, J. \& Desplan, C. Glia relay differentiation cues to coordinate neuronal development in Drosophila. Science 357, 886-891 (2017).

19.Serrano-Saiz, E., Leyva-Díaz, E., De La Cruz, E. \& Hobert, O. BRN3-type POU Homeobox Genes Maintain the Identity of Mature Postmitotic Neurons in Nematodes and Mice. Curr. Biol. 28, 2813-2823.e2 (2018).

20.Deneris, E. S. \& Hobert, O. Maintenance of postmitotic neuronal cell identity. Nat. Neurosci. 17, 899-907 (2014).

21.Leyva-Díaz, E. \& Hobert, O. Transcription factor autoregulation is required for acquisition and maintenance of neuronal identity. Development 146, dev177378 (2019).

22.Xu, C. et al. Control of Synaptic Specificity by Establishing a Relative Preference for Synaptic Partners. Neuron 103, 865-877.e7 (2019).

23. Chen, Y. et al. Cell-type-Specific Labeling of Synapses In Vivo through Synaptic Tagging with Recombination. Neuron 81, 280-293 (2014).

24.Hasegawa, E., Kaido, M., Takayama, R. \& Sato, M. Brain-specific-homeobox is required for the specification of neuronal types in the Drosophila optic lobe. Dev. Biol. 377, 90-99 (2013).

25. Rivera-Alba, M. et al. Wiring Economy and Volume Exclusion Determine Neuronal Placement in the Drosophila Brain. Curr. Biol. 21, 2000-2005 (2011).

26. Meinertzhagen, I. A. \& O’Neil, S. D. Synaptic organization of columnar elements in the lamina of the wild type inDrosophila melanogaster. J. Comp. Neurol. 305, 232-263 (1991).

27.Joesch, M., Schnell, B., Raghu, S. V., Reiff, D. F. \& Borst, A. ON and OFF pathways in Drosophila motion vision. Nature 468, 300-304 (2010). 
28.Fisher, Y. E. et al. A Class of Visual Neurons with Wide-Field Properties Is Required for Local Motion Detection. Curr. Biol. 25, 3178-3189 (2015).

29.Tuthill, J. C., Nern, A., Holtz, S. L., Rubin, G. M. \& Reiser, M. B. Contributions of the 12 Neuron Classes in the Fly Lamina to Motion Vision. Neuron 79, 128-140 (2013).

30.Bahl, A., Serbe, E., Meier, M., Ammer, G. \& Borst, A. Neural Mechanisms for Drosophila Contrast Vision. Neuron 88, 1240-1252 (2015).

31.Lee, W. B. et al. Ocular findings in Jacobsen syndrome. J. AAPOS Off. Publ. Am. Assoc. Pediatr. Ophthalmol. Strabismus 8, 141-145 (2004).

32.Coldren, C. D. et al. Chromosomal microarray mapping suggests a role for BSX and Neurogranin in neurocognitive and behavioral defects in the 11q terminal deletion disorder (Jacobsen syndrome). neurogenetics 10, 89 (2008). 